\title{
HYPEREOISNOPHILIC SYNDROME: A CASE REPORT
}

Satya Srinivas Appalaํ, Sudharsan², Harika ${ }^{3}$, Chandra Sudher ${ }^{4}$, Ramesh $^{5}$

\section{HOW TO CITE THIS ARTICLE:}

Satya Srinivas Appala, Sudharsan, Harika, Chandra Sudher, Ramesh. "Hypereoisnophilic Syndrome: A Case Report". Journal of Evolution of Medical and Dental Sciences 2014; Vol. 3, Issue 23, June 09; Page: 6509-6512, DOI: $10.14260 /$ jemds/2014/2773

ABSTRACT: We report a 28years old male presenting with syncope. A thorough clinical evaluation directed us towards cardiac syncope. Doppler echocardiographic study was used as a main modality of diagnosis and cardiac catheterization confirmed the diagnosis of restrictive cardiomyopathy. We express the contribution of clinical findings and appropriate diagnostic measures in approaching a rare case of systemic eosinophilic syndrome, presenting as restrictive cardiomyopathy.

KEYWORDS: Hypereoisophilia, cardiomyopathy, eosinophillic gastritis.

INTRODUCTION: The hypereosinophilic syndrome involves several organ systems beyond the heart, including the lungs, brain and bone marrow. Both chambers of the heart are involved and show endocardial thickening of the inflow regions and ventricular apices.[1] On histological examination, there are variable degrees of eosinophilic myocarditis of the myocardium and subendocardium, thrombosis and inflammation of small intraluminal coronary vessels, mural thrombosis containing eosinophils and endocardial thickening of several millimeters.

CASE HISTORY: A 28years old male presented to the out-patient department with breathlessness on exertion, fatigability, pedal edema and epigastric pain since 2years, aggravated since last 2 months. O/E- Pulse rate 88/min, irregularly irregular low volume. Blood pressure 100/70 mm Hg, respiratory rate $18 / \mathrm{min}$, bilateral pedal edema present, jugular venous pressure (JVP) raised $(10 \mathrm{~cm} \mathrm{H} 20)$ with prominent " $y$ " descent. CVS examination revealed apical impulse well defined in left fifth intercostal space $1 \mathrm{~cm}$ lateral to mid clavicular line, variable S1, loud P2, systolic murmurs in mitral and tricuspid areas.

Respiratory system revealed clinically no abnormalities. Per abdomen showed congestive hepatomegaly with ascites. On investigations, ECG showed normal voltage complexes with atrial fibrillation, right axis deviation with ST-T changes (reverse tick sign) (Fig. 1). Chest $\mathrm{x}$ ray showed enlarged cardiac silhoute with pulmonary congestion.

Echocardiography showed marked biatrial enlargement, (fig. 2) mildly hypertrophied but normal sized ventricles, moderate MR, severe TR, mild PAH (SPAP $40 \mathrm{mmHg}$ ), fair biventricular function, no intracavitatory clots, with minimal pericardial effusion. Echocardiography measurements LA 64mms, IVS 10 mms, LVID(d) 45mms, LVID(s) 35mms, PWD 8 mms, EF 50\% (fig. 3).

Cardiac catheterization revealed grade III MR, severe TR severe PAH, mild RV systolic dysfunction, no apical obliteration, no endocardial calcification, fair LV systolic dysfunction with normal coronaries. On routine investigation absolute eosinophilic count is 3000per/cumm. Upper gastro intestinal endoscopy revealed multiple ulcers and biopsy has been done, which is showing eosinophilic gastritis (fig. 4). 
DISCUSSION: This patient of heart failure presented with symptoms of systemic and pulmonary congestion was evaluated and significance was given to the raised JVP with prominent " $y$ " decent suggestive of restrictive heart disease. Clinical signs of mitral regurgitation (MR), tricuspid regurgitation (TR) and pulmonary arterial hypertension (PAH) with atrial fibrillation (AF) were significant.

ECG showed normal QRS voltage with AF, right axis deviation and ST-T changes (suggestive of digoxin toxicity). Presence of AF with negative " $y$ " descent made us to evaluate regarding restrictive right heart diseases.[2]

Echocardiography showed biatrial dilatation, restrictive Doppler flows, dilated inferior vena cava, preserved systolic function and atrio ventricular regurgitations which were characteristic of RCM. Echocardiography also helped us in excluding endomyocardial involvement as seen in endomyocardial fibrosis by the absence of obliteration of ventricular cavities and right ventricular outflow tract dilatation. [3]

This was confirmed by cardiac catheterization which revealed markedly elevated ventricular filling pressure with characteristic restrictive haemodynamic pattern and no obliteration of cavities.

Endomyocardial biopsy was not performed as studies have shown its limitation reserved in excluding infiltrative disorders and being a blind procedure ${ }^{[4]}$ Loffler endocarditis is more aggressive and rapidly progresses, affects mainly males and is associated with hypereosinophilia, thromboemboli, systemic arteritis; endomyocardial fibrosis occurs in a younger distribution, affects young children and is only variably associated with eosinophilia.[5]

In temperate climates, endomyocardial disease is closely associated with significant hypereosinophilia, which can have several different causes. Hypereosinophilia associated with Loffler endocarditis usually is characterized by eosinophil counts exceeding 1500/cumm for at least 6 months. Most patients with this degree of hypereosinophilia will have cardiac involvement.

The eosinophilia may be secondary to leukemia, reactive disorders such as parasite infection, allergies, granulomatous syndromes, hypersensitivity, or neoplastic disorders. The mechanism by which eosinophils participate in the development of cardiac disease remains incompletely understood. These cells have the capacity to directly infiltrate tissues or to release factors that may exert toxicity.

The observation that patients with Loffler endocarditis have degranulated eosinophils in their peripheral blood supports the idea that these granules contain cardiotoxic substances, capable of causing the necrotic phase of endomyocardial disease, which leads to the thrombotic and fibrotic phases once the eosinophilia resolves. ${ }^{[6]}$

Here we are reporting a case of hypereosinophilic syndrome presenting as restrictive cardiomyopathy and eosinophilic gastritis, a high suspicion should be kept in mind for the above diagnosis when patient presents with hypereosinophilia. Eosinophilic gastritis is a rare and heterogeneous condition characterized by patchy or diffuse eosinophilic infiltration of gastrointestinal tissue; presentation may vary depending on location as well as depth and extent of bowel wall involvement. It usually runs a chronic relapsing course.[7]

Antiplatelets, digoxin, warfarin, Corticosteroids mainstay of treatment. 


\section{REFERENCES:}

1. Hoit BD, Miller D. Restrictive, obliterative and infiltrative cardiomyopathy: restrictive cardiomyopathy. Hurst Heart. 2004; 78: 1937.

2. Ammash, NM, Seward JB, Bailey KR et al. Clinical profile and outcome of idiopathic restrictive cardiomyopathy. Circulation 2000; 101: 2490-2496.

3. Nair DN. Endomyocardial fibrosis in Kerala. Indian Heart J 1982; 34: 412- 7.

4. Wynne J, Braunwald E. Cardiomyopathy and myocarditis: restrictive cardiomyopathy. Harrison's internal medicine. 2011; 231: 1485.

5. Appleton CP, Hatle LK, Popp RL. Relation of transmitral flow velocity patterns to left ventricular diastolic function: new insights from a combined hemodynamic and Doppler echocardiographic study. J Am Coll Cardiol. 1988; 12: 426-440.

6. Seth S, Thatai D, Sharma S, et al. Clinico-pathological evaluation of restrictive cardiomyopathy (endomyocardial fibrosis and idiopathic restrictive cardiomyopathy) in India. Eur J Heart Failure 6 (20 04); 723- 729.

7. Klein A, Cohen G, Pietronungo J et al. Differentiation of constrictive pericarditis from restrictive cardiomyopathy by Doppler transesophageal echocardiographic measurements of respiratory variation in pulmonary venous flow. J Am Coll Cardiol 1993; 22: 1935-1943.

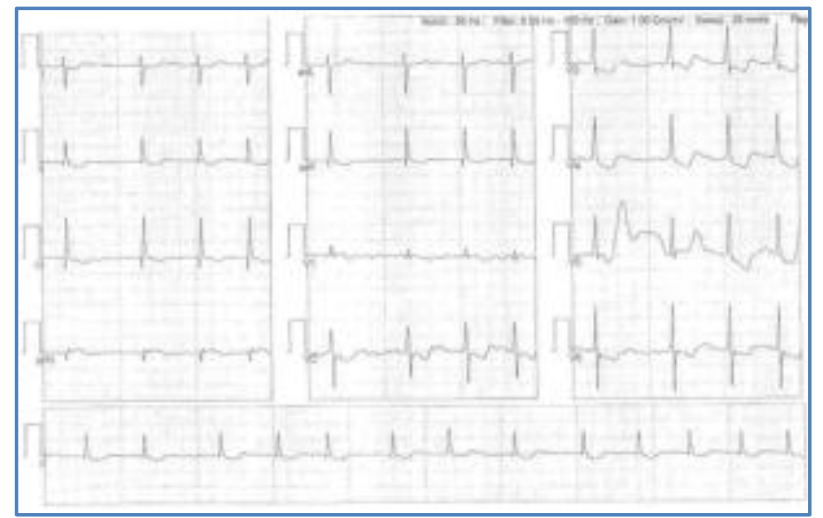

Fig 1: ECG atrial fibrillation

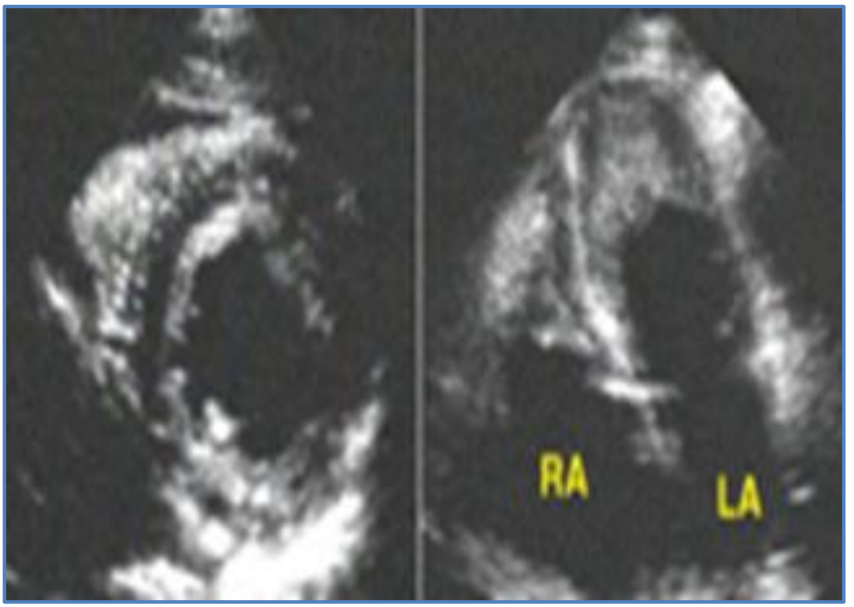

Fig 2 : 2d echo showing bi atrial enlargement with TR 


\section{CASE REPORT}

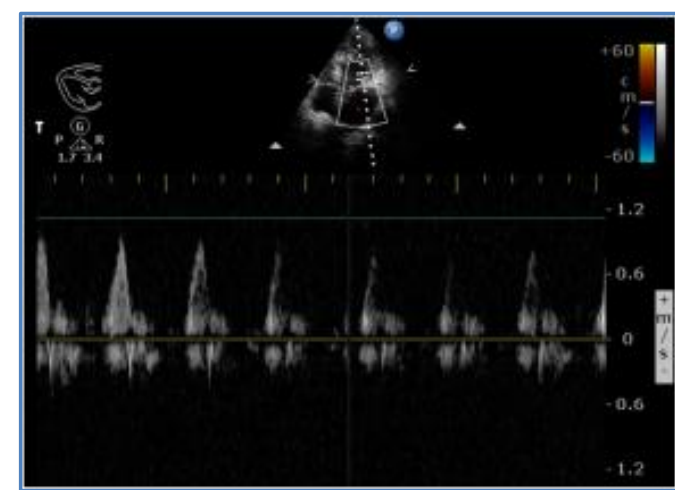

Fig 3 : 2d echo showing bi atrial enlargement with TR

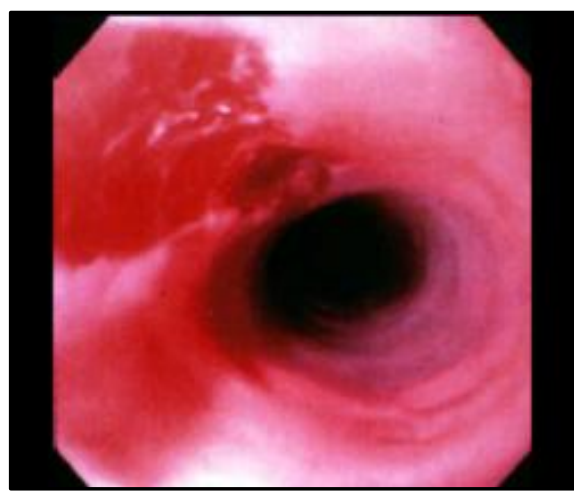

Fig 4: Upper g.i endoscopy showing nonhealing ulcers

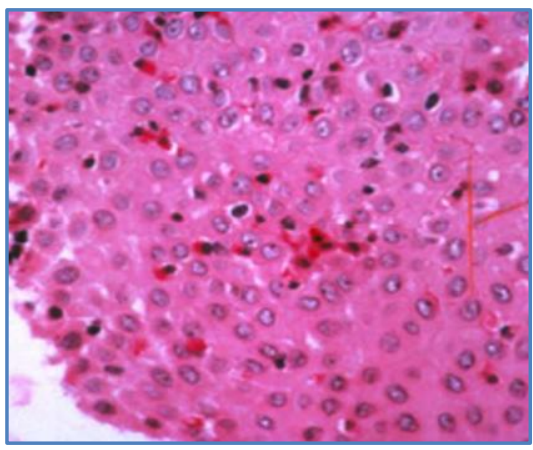

Fig 5: biopy showing esinophillic gastritis

\section{AUTHORS:}

1. Satya Srinivas Appala

2. Sudharsan

3. Harika

4. Chandra Sudher

5. Ramesh

\section{PARTICULARS OF CONTRIBUTORS:}

1. Assistant Professor, Department of Internal Medicine, Kamineni Institute of Medical Sciences, Narketpally, Andhra Pradesh.

2. Professor, Department of Internal Medicine, Kamineni Institute of Medical Sciences, Narketpally, Andhra Pradesh.

3. Post Graduate, Department of Internal Medicine, Kamineni Institute of Medical Sciences, Narketpally, Andhra Pradesh.

4. Post Graduate, Department of Internal Medicine, Kamineni Institute of Medical Sciences, Narketpally, Andhra Pradesh.
5. Post Graduate, Department of Internal Medicine, Kamineni Institute of Medical Sciences, Narketpally, Andhra Pradesh.

\section{NAME ADDRESS EMAIL ID OF THE CORRESPONDING AUTHOR:}

Dr. Satya Srinivas Appala,

Flat No. 12,

Prashanthi Apartments, \#1, 6/1, Arundel Peta, Guntur-522002,

Andhra Pradesh.

Email: drsujaym@gmail.com

Date of Submission: 01/05/2014. Date of Peer Review: 02/05/2014.

Date of Acceptance: 09/05/2014.

Date of Publishing: 07/06/2014. 